\title{
Shunting outcomes in posthemorrhagic hydrocephalus: results of a Hydrocephalus Clinical Research Network prospective cohort study
}

\author{
John C. Wellons III, MD, MSPH, ${ }^{1}$ Chevis N. Shannon, MPH, MBA, DrPH, ${ }^{1}$ Richard Holubkov, PhD, ${ }^{2}$ \\ Jay Riva-Cambrin, MD, MSc, ${ }^{3}$ Abhaya V. Kulkarni, MD, PhD, ${ }^{4}$ David D. Limbrick Jr., MD, PhD, ${ }^{5}$ \\ William Whitehead, MD, MPH, ${ }^{6}$ Samuel Browd, MD, PhD, ${ }^{7}$ Curtis Rozzelle, MD, ${ }^{8}$ \\ Tamara D. Simon, MD, MSPH, ${ }^{9}$ Mandeep S. Tamber, MD, PhD, ${ }^{10}$ W. Jerry Oakes, MD, ${ }^{8}$ \\ James Drake, MBBCh, MSc, ${ }^{4}$ Thomas G. Luerssen, MD, ${ }^{6}$ and John Kestle, MD, MSc, ${ }^{11}$ \\ for the Hydrocephalus Clinical Research Network
}

1Department of Neurosurgery, Vanderbilt University Medical Center, Nashville, Tennessee; ${ }^{2}$ Data Coordinating Center, University of Utah, Salt Lake City, Utah; ${ }^{3}$ Division of Neurosurgery, University of Calgary, Alberta, Canada; ${ }^{4}$ Department of Neurosurgery, University of Toronto, Ontario, Canada; ${ }^{5}$ Department of Neurosurgery, Washington University St. Louis, Missouri; ${ }^{6}$ Department of Neurosurgery, Baylor College of Medicine, Houston, Texas; ${ }^{7}$ Department of Neurosurgery, University of Washington Medical Center, Seattle, Washington; ${ }^{8}$ Department of Neurosurgery, University of Alabama-Birmingham, Alabama; ${ }^{9}$ Department of Pediatrics, University of Washington Medical Center, Seattle, Washington; ${ }^{10}$ Department of Neurosurgery, University of Pittsburgh Medical Center, Pittsburgh, Pennsylvania; and ${ }^{11}$ Department of Neurosurgery, University of Utah, Salt Lake City, Utah

OBJECTIVE Previous Hydrocephalus Clinical Research Network (HCRN) retrospective studies have shown a $15 \%$ difference in rates of conversion to permanent shunts with the use of ventriculosubgaleal shunts (VSGSs) versus ventricular reservoirs (VRs) as temporization procedures in the treatment of hydrocephalus due to high-grade intraventricular hemorrhage (IVH) of prematurity. Further research in the same study line revealed a strong influence of center-specific decision-making on shunt outcomes. The primary goal of this prospective study was to standardize decision-making across centers to determine true procedural superiority, if any, of VSGS versus VR as a temporization procedure in highgrade IVH of prematurity.

METHODS The HCRN conducted a prospective cohort study across 6 centers with an approximate 1.5- to 3-year accrual period (depending on center) followed by 6 months of follow-up. Infants with premature birth, who weighed less than $1500 \mathrm{~g}$, had Grade 3 or 4 IVH of prematurity, and had more than 72 hours of life expectancy were included in the study. Based on a priori consensus, decisions were standardized regarding the timing of initial surgical treatment, upfront shunt versus temporization procedure (VR or VSGS), and when to convert a VR or VSGS to a permanent shunt. Physical examination assessment and surgical technique were also standardized. The primary outcome was the proportion of infants who underwent conversion to a permanent shunt. The major secondary outcomes of interest included infection and other complication rates.

RESULTS One hundred forty-five premature infants were enrolled and met criteria for analysis. Using the standardized decision rubrics, 28 infants never reached the threshold for treatment, 11 initially received permanent shunts, 4 were initially treated with endoscopic third ventriculostomy (ETV), and 102 underwent a temporization procedure (36 with VSGSs and 66 with VRs). The 2 temporization cohorts were similar in terms of sex, race, IVH grade, head (orbitofrontal) circumference, and ventricular size at temporization. There were statistically significant differences noted between groups in gestational age, birth weight, and bilaterality of clot burden that were controlled for in post hoc analysis. By Kaplan-Meier analysis, the 180-day rates of conversion to permanent shunts were $63.5 \%$ for VSGS and $74.0 \%$ for VR $(p=0.36$, log-rank test). The infection rate for VSGS was $14 \%(5 / 36)$ and for VR was $17 \%(11 / 66 ; p=0.71)$. The overall compliance rate with the standardized decision rubrics was noted to be $90 \%$ for all surgeons.

ABBREVIATIONS BSID-III = Bailey Standardized Infant Development-III; Cl = confidence interval; DCC = Data Coordinating Center; ETV = endoscopic third ventriculostomy; FOHR = frontal occipital horn ratio; HCRN = Hydrocephalus Clinical Research Network; IVH = intraventricular hemorrhage; OFC = orbitofrontal circumference; PHH = posthemorrhagic hydrocephalus; SOPHH = shunting outcomes in posthemorrhagic hydrocephalus; VP = ventriculoperitoneal; VR = ventricular reservoir; VSGS = ventriculosubgaleal shunts.

SUBMITTED September 7, 2016. ACCEPTED January 26, 2017.

INCLUDE WHEN CITING Published online April 28, 2017; DOI: 10.3171/2017.1.PEDS16496. 
CONCLUSIONS A standardized protocol was instituted across all centers of the HCRN. Compliance was high. Choice of temporization techniques in premature infants with IVH does not appear to influence rates of conversion to permanent ventricular CSF diversion. Once management decisions and surgical techniques are standardized across HCRN sites, thus minimizing center effect, the observed difference in conversion rates between VSGSs and VRs is mitigated.

https://thejns.org/doi/abs/10.3171/2017.1.PEDS16496

KEY WORDS hydrocephalus; intraventricular hemorrhage; prematurity; ventricular shunt; ventriculosubgaleal shunt; ventricular reservoir; Hydrocephalus Clinical Research Network

$\mathrm{H}$ YDROCEPHALUS arising from Grade 3 or 4 intraventricular hemorrhage (IVH) of prematurity (i.e., posthemorrhagic hydrocephalus $[\mathrm{PHH}]$ ) continues to affect (conservatively) up to 3000 infants in the US yearly. ${ }^{1,15}$ In reporting the findings of a study performed by the Neonatal Research Network, funded by the Eunice Kennedy Shriver National Institute of Child Health and Human Development, Adams-Chapman et al. reported worsened neurodevelopmental outcomes in very low birth weight and extremely low birth weight infants with Grade 3 or $4 \mathrm{PHH}$ and a ventricular shunt versus the same categories of children without a ventricular shunt. ${ }^{1}$ The question that then arises is if any of several means of early hydrocephalus surgical management can impact the ultimate need to place a permanent shunt. Several single-center series report the incidence of shunt placement in these children using either subcutaneous reservoirs for intermittent percutaneous removal of CSF or ventriculosubgaleal shunt (VSGS) placement. ${ }^{3,6-9,11,16,17,19,22,23,25-27}$ Two recent notable publications report the retrospectively reviewed single-center experience using both..$^{14,28}$ In 2009, the Hydrocephalus Clinical Research Network (HCRN) published an initial paper in which the permanent shunt rates were evaluated in a 147-patient retrospective nonstandardized cohort. Use of a ventricular reservoir (VR) led to a lower rate of permanent shunt placement in a statistically significant fashion. ${ }^{30}$ However, institutional bias as denoted by "center effect" was noted in subsequent publications. ${ }^{24}$ Therefore, the HCRN developed standardized management rubrics that were based on findings in the prior 2 studies ${ }^{24,30}$ and agreed upon by consensus. This prospective study evaluated the network experience implementing these standardized management rubrics using both VR placement for intermittent CSF removal (VR group) and VSGS placement for the treatment of PHH due to Grade 3 or 4 IVH (VSGS group). The primary goal of this study was to identify procedural superiority, if it existed, between VRs and VSGSs, once decision-making is standardized.

\section{Methods}

\section{Study Population and Design}

Appropriate IRB approval was obtained at the University of Alabama-Birmingham as the Clinical Coordinating Center and as a participating center. The study was performed under IRB approval at the University of Utah Data Coordinating Center (DCC); IRB approval was then obtained at the 6 remaining HCRN sites at the time of study initiation. These 7 sites included Primary Children's Hospital, University of Utah; Children's Hospital of Alabama, University of Alabama at Birmingham;
Hospital for Sick Children, University of Toronto; Texas Children's Hospital, Baylor College of Medicine; Seattle Children's Hospital, University of Washington; Children's Hospital of Pittsburgh, University of Pittsburgh; and St. Louis Children's Hospital, Washington University in St. Louis. Due to very low accrual (1 patient), 1 center did not renew IRB approval, thus voiding any relevant follow-up. Therefore, data from a total of 6 centers were used in the final analysis.

The study was designed as a multicenter prospective observational study to initially enroll patients over an 18-month period. Infants were not randomized between VRs and VSGSs. The decision of which temporization procedure to assign was left to the surgeon. The study was initiated in a staggered fashion across the 6 participating centers of the network. However, at all centers, enrollment was extended for up to 39 months until patient accrual was halted across the network in preparation for analysis. Data were collected on paper study forms, transformed to a centralized database (the trial database) maintained at the University of Utah DCC, and cleaned and analyzed at the end of the study in preparation for abstract submission and then for ultimate manuscript preparation. ${ }^{2,18}$ Inclusion criteria were very low birth weight $(<1500 \mathrm{~g})$ premature infants with Grade 3 or 4 IVH. Exclusion criteria were infants who were judged to have less than 72 hours to live due to other non-hydrocephalus-related conditions. Patients were managed per previously agreed upon rubrics: "Decision to Treat," "Decision to Shunt versus Temporize," "Decision to Convert (VR) to Permanent Shunt," and "Decision to Convert (VSGS) to Permanent Shunt" (Figs. 1-4). Relevant rubric definitions including clot size and density were agreed upon as well (Table 1). Each rubric was designed using data taken from a previous HCRN study as well as consensus among the HCRN investigatorsurgeons. ${ }^{24}$ Only partial consensus could be obtained on the infant weight at which to either temporize or permanently place a shunt. Therefore, a surgeon preference decision node was engineered into the appropriate rubrics to inform later protocols.

\section{Outcome Measures}

The primary outcome was conversion of either a VR or VSGS to a permanent shunt within 6 months of the temporization procedure. Secondary outcomes for the purposes of the primary study were the rates of complications, including infection, CSF leak, death, or new intracranial hemorrhage. As the patients within the cohort reach 18-24 months of corrected age, the Bailey Standardized Infant Development-III (BSID-III) test is currently being performed at each center according to local protocols, scored, and recorded in a DCC-centralized database. Once com- 


\section{BW $<1500 \mathrm{~g}$ \\ Grade III or IV IVH \\ $>72$ hour life expectancy \\ $\mathrm{FOHR} \geq 0.50$}

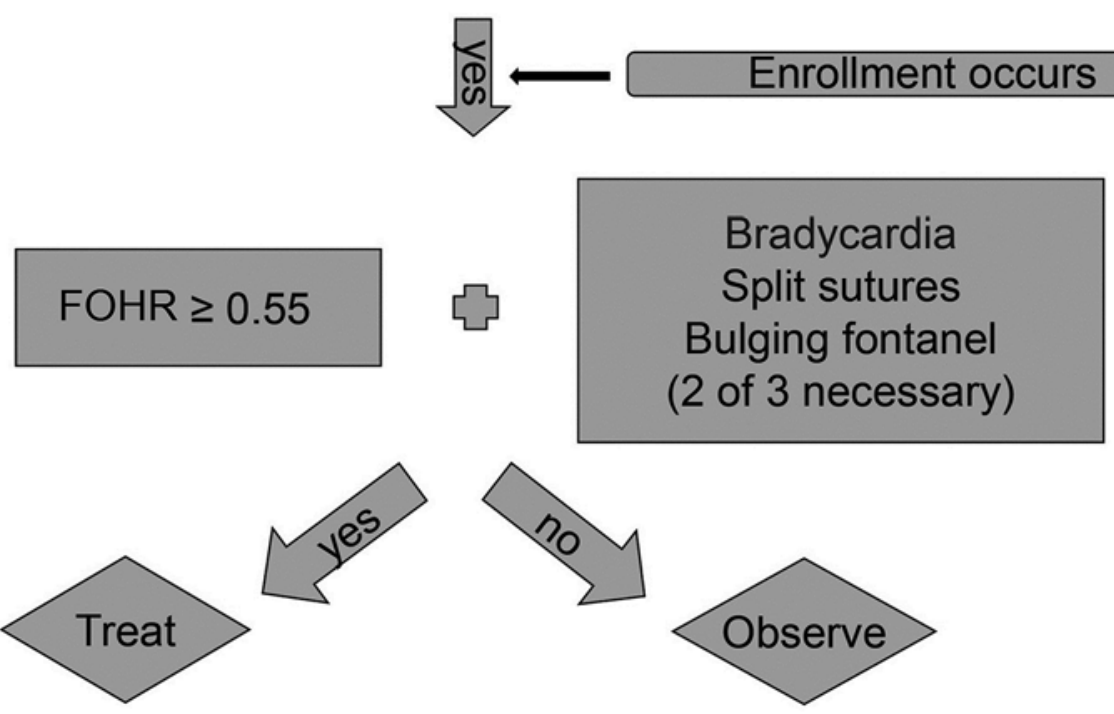

FIG. 1. Rubric illustrating the decision to treat. BW = body weight.

pleted, results of this secondary outcome will be reported in a second paper.

\section{Study Compliance}

Compliance with the decision rubrics was evaluated overall, and subdivided into 2 groups: HCRN surgeon- investigators and HCRN surgeon-noninvestigators (those surgeons who are part of an HCRN center, who have agreed to standardize, but who do not have a formal role within the HCRN). Compliance was determined by 2 methods. For enrollment, the frontal occipital horn ratio (FOHR) and patient characteristics were reviewed from

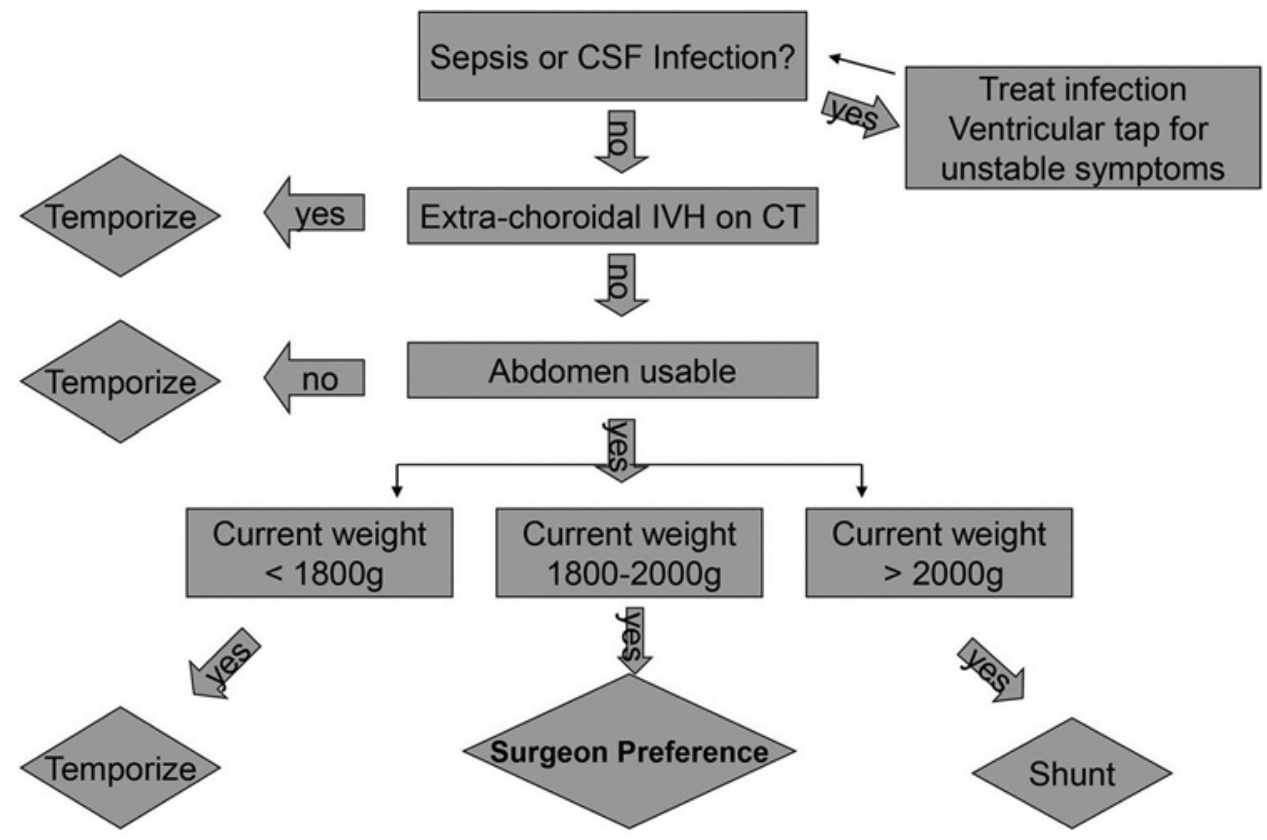

FIG. 2. Decision rubric regarding whether to temporize or place a shunt. 

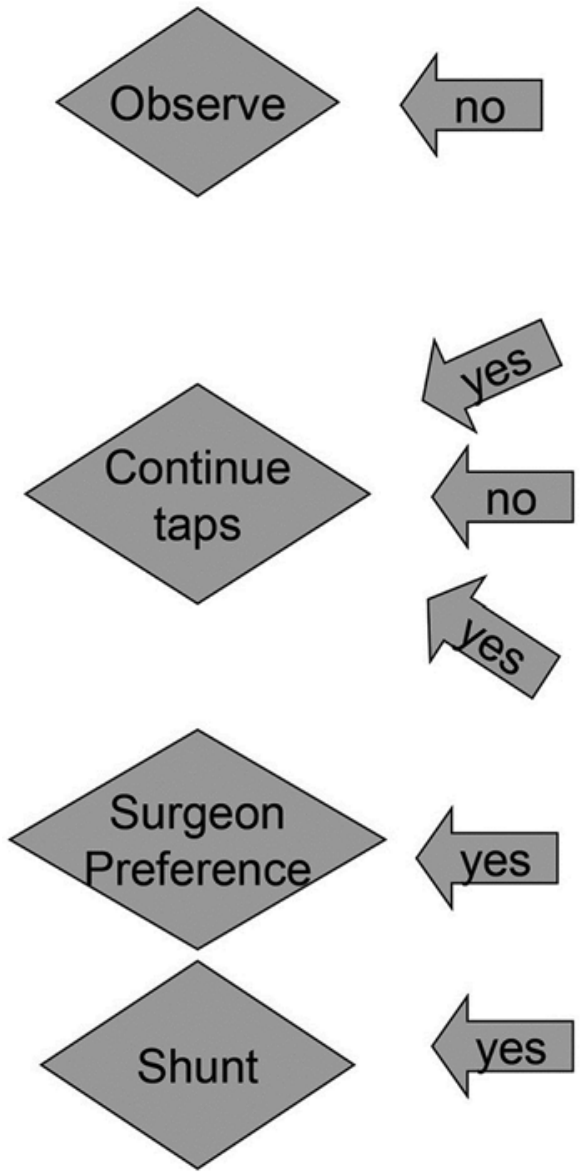

FIG. 3. Decision rubric illustrating whether to convert a VR.

the prospective database. For temporization and conversion, compliance was determined at the time of data recording on the data sheet by the site research coordinator. Unclear violations or adherence episodes were identified by the site research coordinators and adjudicated by the study and DCC primary investigators. During the study, endoscopic third ventriculostomy (ETV) became more used. Four patients were initially treated with ETV, and 1 VSGS was converted to permanent CSF diversion using an ETV. For the purpose of this study, ETV was considered compliant if chosen according to the rubrics, and for the purpose of the primary outcome falls under the terminology "shunt."

In addition to standardization of decision-making among surgeons, the VR and VSGS procedures themselves were standardized. Surgical checklists (Online Appendix) and videos were created and circulated among the investigator and noninvestigator surgeons of the HCRN. The procedure for intermittent removal of CSF from VRs was also standardized, including amount per "tap" and the clinical reasons for removal of CSF (Online Appendix).

\section{Statistical Analysis}

Binary and categorical characteristics were compared between temporization cohorts using Pearson's chi-square test or Fisher's exact test when the expected number of values within any cell was under 5 . Continuous characteris-
$\mathrm{FOHR} \geq 0.55$ and

Persistent tapping by term date

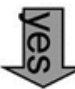

\section{Extra-choroidal IVH}

局

Abdomen usable

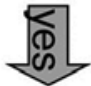

Current weight $<1800 \mathrm{~g}$

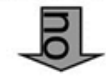

Current weight 1800-2000g

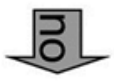

\section{Current weight $\geq 2000 \mathrm{~g}$}

tics were compared between cohorts using t-tests. Curves of freedom from permanent shunting over time were constructed using the Kaplan-Meier approach and compared via the log-rank test. The Cox proportional hazards model was used to compute hazard ratios for conversion to permanent shunting, while adjusting for potential confounding factors between the 2 cohorts. All analyses were performed using SAS (version 9.4, SAS Institute, Inc.).

\section{Results}

Enrollment across all 6 centers is detailed in Fig. 5. There were 145 premature infants enrolled across the network. According to the standardized decision rubrics, 28 never reached the threshold for treatment, 11 initially received a permanent shunt, 4 were initially treated with ETV, and 102 underwent a temporization procedure (36 with a VSGS and 66 with a VR). Analyses for this study were restricted to the 102 infants who underwent temporization.

The 2 temporization cohorts were similar in terms of sex, race, IVH grade, head orbitofrontal circumference (OFC), and ventricular size at temporization (Table 2). There were 3 relevant statistically significant differences noted between the 2 groups. The VSGS cohort had significantly lower gestational age at birth and lower corresponding birth weight, while substantially more children in the 


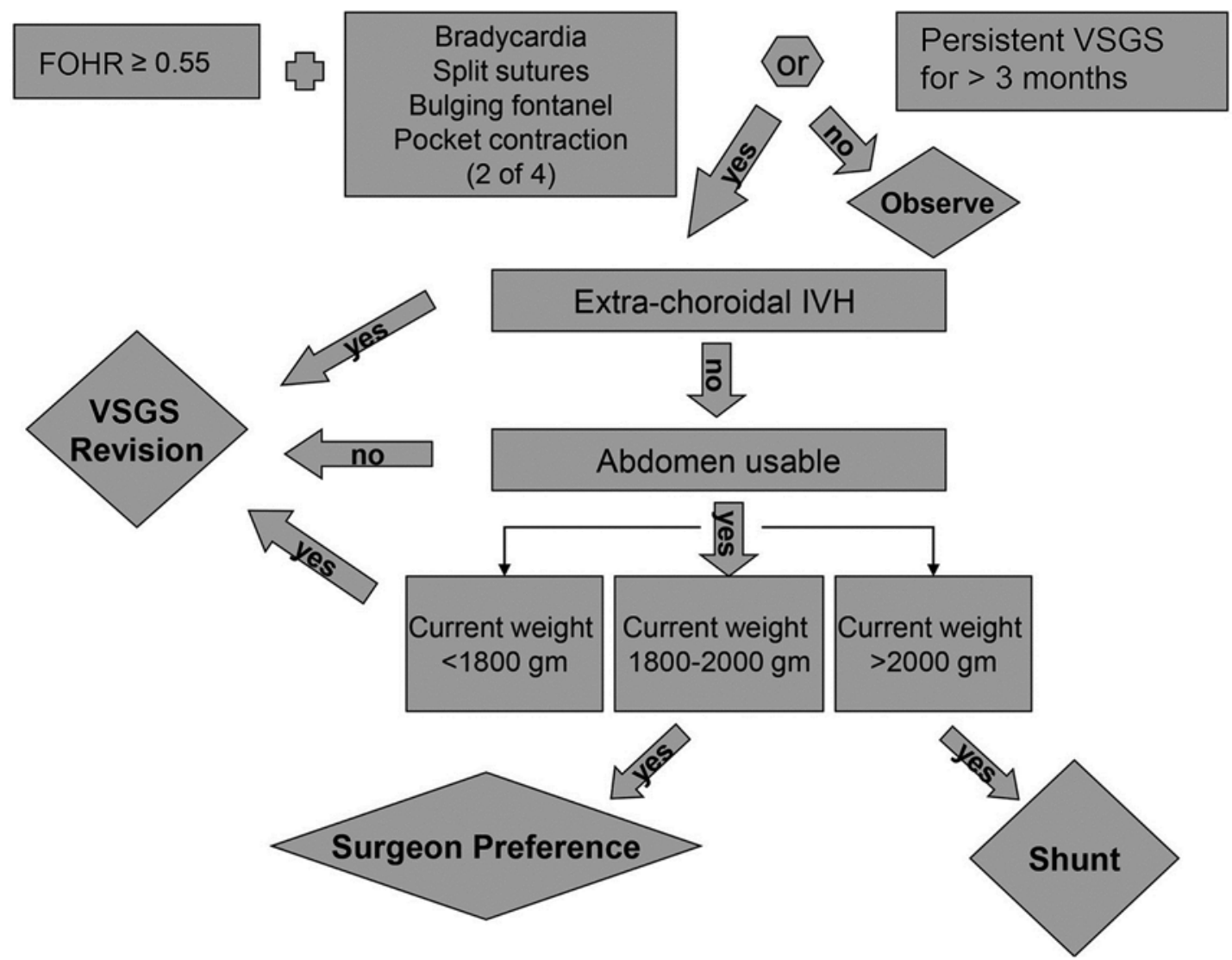

FIG. 4. Decision rubric regarding whether to convert a VSGS. gm = grams.

VR cohort had bilateral clot burden. These differences were controlled for in post hoc analysis.

At 6 months after temporization, 21 of 36 children in the VSGS arm and 45 of 66 in the VR arm had converted to a permanent shunt ( 1 of the 21 VSGS conversions was an ETV). The 180-day conversion rates were $63.5 \%$ for VSGS and $74.0 \%$ for VR; the freedom-from-event curves were not significantly different $(\mathrm{p}=0.36$, log-rank test; Fig. 6). In a Cox proportional hazards model, the unadjusted hazard ratio for a child treated with VSGS to convert to a permanent shunt, relative to a child temporized with a VR, was 0.79 (95\% confidence interval [CI] 0.47-1.32). After adjustment for the factors showing a baseline imbalance between VSGS and VR (gestational age, birth weight, and bilaterality of clot), the hazard ratio was virtually unchanged at 0.75 (95\% CI $0.42-1.34$ ).

The incidence of complications occurring after temporization and before permanent shunting (if this occurred) was not significantly different between groups (Table 3 ). The infection rate for VSGS was 14\% (5/36) and for VR was $17 \%(11 / 66 ; p=0.71)$. Rates of CSF leak differed only by $1 \%$ between cohorts. During the 6 months after tempo-

\section{TABLE 1. Agreed-upon SOPHH definitions}

\begin{tabular}{|c|c|}
\hline $\mathrm{SOPHH}$ & Definition \\
\hline Bradycardia & $\begin{array}{l}\text { Persistent bradycardia was defined for the purposes of the SOPHH study as } 3 \text { or more prolonged episodes in a } 24-\mathrm{hr} \\
\text { period that could not be explained by another medical condition. }\end{array}$ \\
\hline $\begin{array}{l}\text { Split sutures (midparietal } \\
\text { sagittal suture) }\end{array}$ & $\geq 2-m m$ split $(+),<2-m m$ not split (-) \\
\hline Bulging anterior fontanel & Above the level of the surrounding bone $(+)$, at or below the level of the surrounding bone (-) \\
\hline Extrachoroidal IVH & $\begin{array}{l}\text { Density: hyperdense (CT)/hyperechogenic (U/S) (+), iso- or hypodense (CT)/iso- or hyperechogenic (U/S) (-); size (re- } \\
\text { ported in length \& width): } \geq 2 \mathrm{~cm}^{2}(+),<2 \mathrm{~cm}^{2}(-)\end{array}$ \\
\hline Abdomen criteria & $\begin{array}{l}\text { The abdomen is considered unusable in the setting of active necrotizing enterocolitis, an unrepaired omphalocele, unre- } \\
\text { paired bladder extrophy, or any unresolved abdominal issue that would be thought to inhibit CSF absorption \& therefore } \\
\text { permanent shunt function. In rare cases in which no foreseeable resolution for the abdominal issue exists, permanent } \\
\text { shunting may take place through the use of either the pleural space or the right atrium for distal absorption. }\end{array}$ \\
\hline
\end{tabular}

+ = positive; - = negative; $\mathrm{U} / \mathrm{S}=$ ultrasound. 


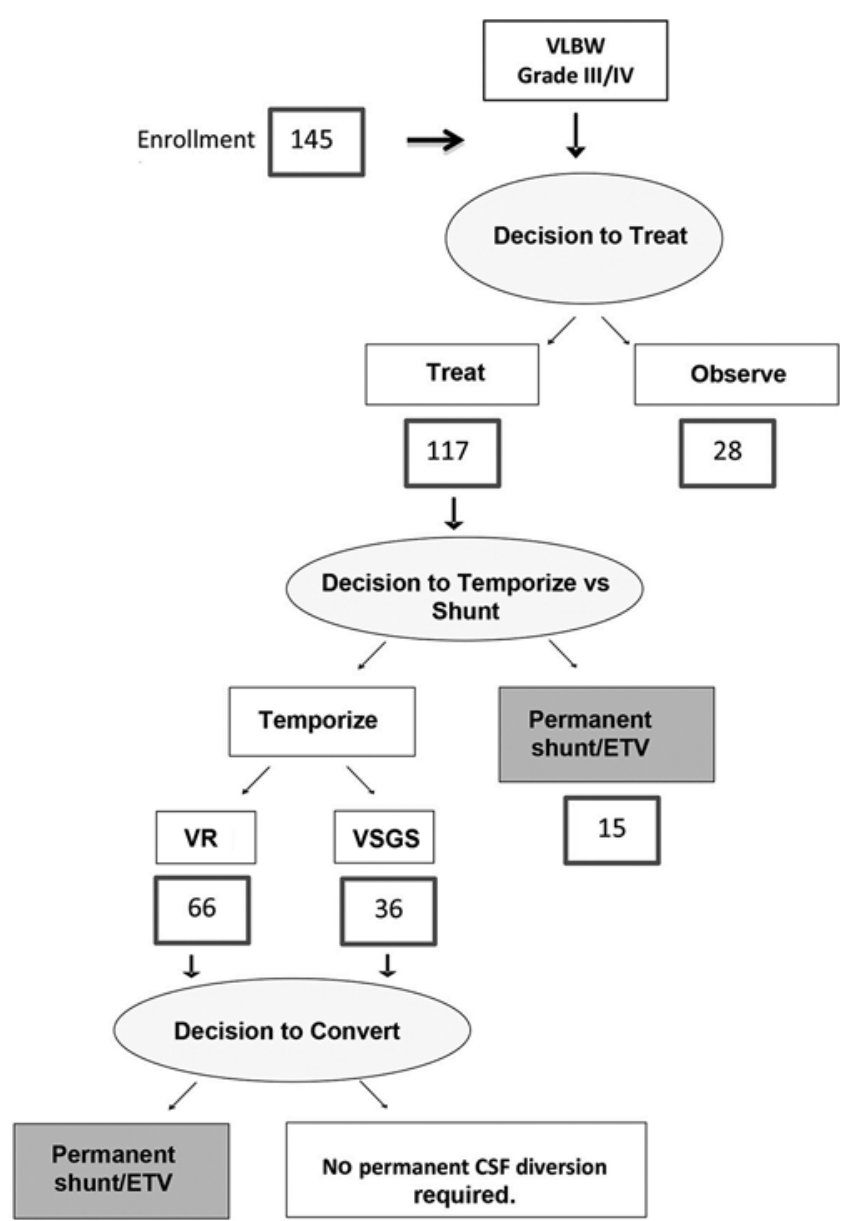

FIG. 5. SOPHH enrollment across the HCRN. VLBW = very low birth weight.

rization, a total of 13 children died (6 with VSGSs and 7 with VRs, $\mathrm{p}=0.54$ using the Fisher's exact test comparing the respective $17 \%$ and $11 \%$ mortality rates).

The overall surgeon compliance rate with the rubrics was $90 \%$. There was a difference between HCRN investigator surgeons and noninvestigator surgeons overall (Table 4). The compliance at enrollment was calculated based on whether the initial FOHR and patient characteristics were appropriate when compared with the protocol. The compliance at temporization and conversion time points was determined by the coordinator at the time of data entry and recorded in a separate field. There appeared to be no statistical difference in compliance with the "Decision to Temporize versus Shunt" or "Decision to Convert" rubrics. There was a statistically significant difference between initial enrollment, however, as HCRN investigator surgeon enrollment compliance was 79/87 (91\%) while noninvestigator surgeon enrollment compliance was 44/58 $(76 \% ; \mathrm{p}=0.014)$. This factor likely drove the overall difference when all 3 time points were combined into a single compliance data set.

\section{Discussion}

The optimal management of PHH is unknown and very little evidence is available to inform decision-making. In premature infants who develop $\mathrm{PHH}$, we have no evidence to guide when in the evolution of $\mathrm{PHH}$ we should treat or how we should treat. The decision of when to intervene has traditionally been based in part on imaging criteria (size of the ventricles, density of the blood clot), the clinical signs and symptoms (including apnea and bradycardia, approximation of the cranial sutures, and status of the anterior fontanel), and/or the head OFC. Across North America and Europe, there is tremendous physician variation in the application of these criteria to determine when to initiate treatment. ${ }^{4}$ Often, due to conditions leading to the inability of the child to tolerate a permanent shunt (i.e., IVH burden, concomitant necrotizing enterocolitis, CSF protein levels, or low relative weight at symptomatic hydrocephalus presentation), the infant may undergo a temporizing surgical procedure to divert CSF while these issues resolve. The overall number of infants in the US who initially undergo surgical temporary CSF diversion rather than permanent shunt placement is difficult to determine. However, published rates of conversion from a temporary device to a permanent shunt in this population range from just over $50 \%$ to as high as $85 \%$., 3,6-9,11,13,16,22,25,27 Traditional temporization approaches include diuretic administration and serial lumbar puncture and have been shown to be largely ineffective in preventing the need for permanent shunting. ${ }^{31,32}$ The patient is therefore typically treated with 1 of 2 available surgical temporization procedures: subcutaneously implanted VRs or VSGSs. These procedures are physiologically very different, and there is little evidence comparing their efficacy or their impact on the incidence of permanent shunting after temporization. The decision between VR and VSGS, therefore, rests almost entirely on physician training or local practice patterns and must be made with virtually no useful data. ${ }^{24}$ A $2011-2012$ survey of the members of the American Society of Pediatric Neurosurgeons (76 members responding) revealed virtual equipoise between the 2 procedures as well as a willingness to change if a relevant difference between them was identified ("Moving Toward Patient-Oriented Outcomes for PHH," American Society of Pediatric Neurosurgeons Meeting, Puerto Rico, 2012).

A subcutaneously implanted VR is technically straightforward and has the advantage of control of CSF drainage and thereby ventricle size. Also, the transcutaneous CSF withdrawal, with incremental removal of blood product and protein from within the ventricles, has been hypothesized to diminish the chance of long-term communicating hydrocephalus. $3,6,7,9,11,16 \mathrm{VR}$ avoids the main complications of other means of intermittent CSF withdrawal, such as repeated lumbar puncture (which frequently yields only a small volume of CSF) and repeated transcortical ventricle puncture (with its risk of intracranial hemorrhage or cystic encephalomalacia). ${ }^{6}$ Proponents of VR also believe that it can reduce the need for permanent ventriculoperitoneal (VP) shunting in some patients by allowing the CSF to build up between taps (thereby challenging the infant's natural CSF absorption system). Complications of the reservoir include CSF leak, infection, or possible skin breakdown, with an incidence up to $22 \% .{ }^{5,11,20,23}$ Some reports suggest that permanent VP shunting can be avoided in 10\%-30\% of cases after VR, but the actual incidence is 
TABLE 2. Patient information according to initial temporization approach

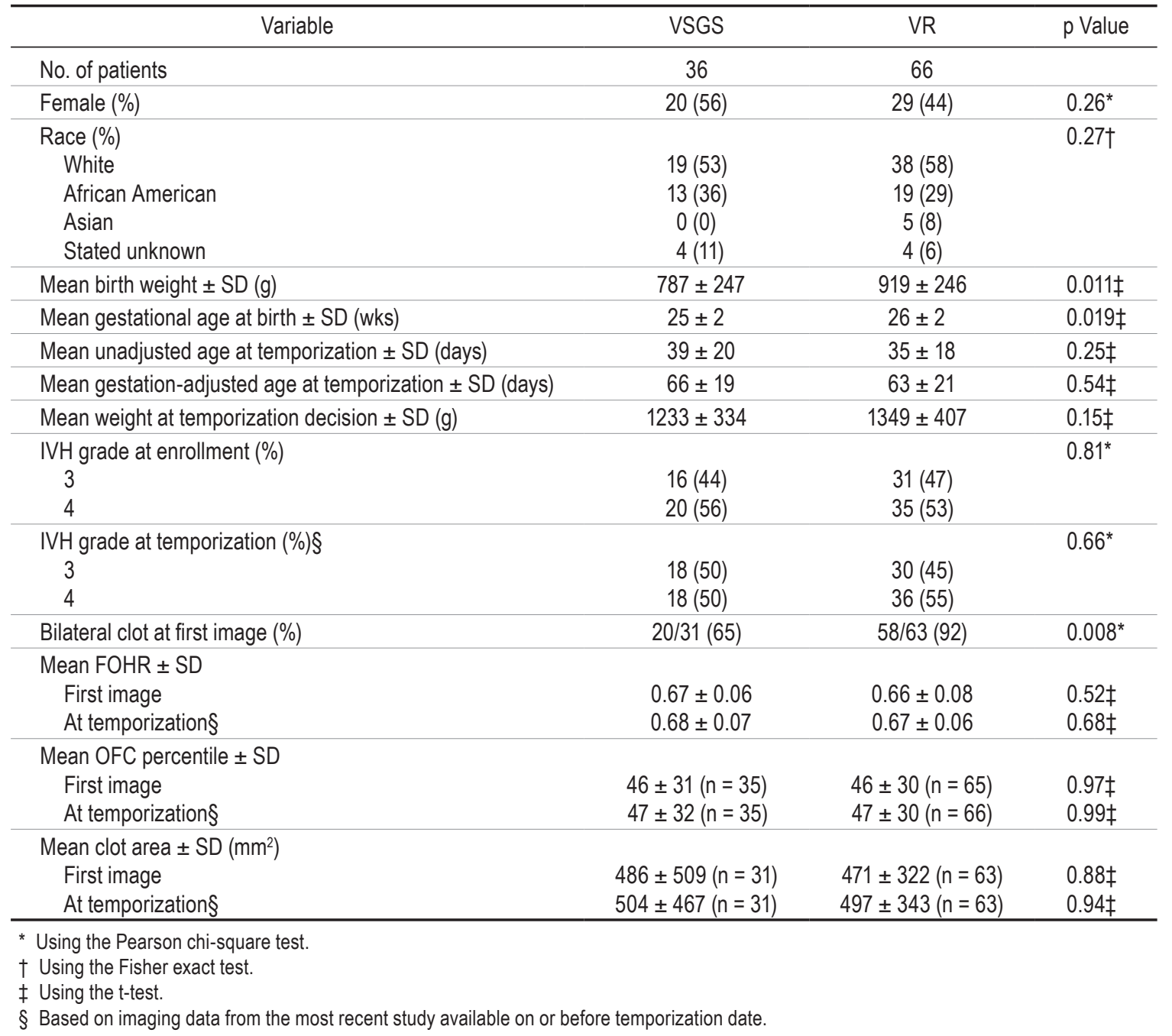

likely related to multiple factors, especially treatment selection criteria (i.e., when to treat, when to convert). ${ }^{7,20,23,33}$

VSGSs have the benefit of a closed system of CSF diversion without the perceived risks associated with intermittent transcutaneous tapping. Proponents of VSGSs argue that its main advantages include maintaining a closed system in which fluid and electrolytes are not lost, maintaining mild back-pressure from the subgaleal space that challenges the natural CSF absorptive pathways to "kick start" normal function, and the potential for earlier discharge home in lieu of continued hospitalization for tapping and electrolyte management. An initial VSGS typically functions for an average of 37 days and a revised VSGS for about 32 days after the first revision. ${ }^{27}$ Some investigators have reported the avoidance of VP shunting in $20 \%$ of 23 patients following VSGS, but again, this outcome was influenced by multiple factors, including treatment selection criteria as discussed above. . $22,25,26^{2}$

An early HCRN retrospective cohort study designed to evaluate historical conversion of VRs and VSGSs to a permanent shunt across the network included 147 infants who underwent surgical intervention for PHH. Of these, 127 $(86 \%)$ were initially treated using a temporizing mecha- nism (VR or VSGS) with 20 (14\%) treated initially with a permanent CSF shunt. The proportions of patients undergoing permanent shunt placement after VSGS and VR were $31 / 36(86 \%)$ and $61 / 88(69 \%)$, respectively $(p=0.05)$. This was one of the first and largest studies to directly compare the outcomes of these 2 interventions. ${ }^{30}$ Since the initial study was published, 2 single-center studies from St. Louis Children's Hospital and from Johns Hopkins University compared VR versus VSGS use, and neither study showed a significant difference between the 2 procedures in terms of need for ultimate permanent shunt placement. ${ }^{14,28}$

During internal discussions of decision-making for these patients, there was a concern regarding potential confounding factors from center to center. Due to this concern, a second multicenter study was conducted by the HCRN to determine the factors influencing when an infant with $\mathrm{PHH}$ received a temporization procedure (VR or VSGS) versus a permanent shunt and when they were converted from a temporary shunt to a permanent shunt. A total of 110 infants were included in this retrospective study. Only larger ventricle size $(\mathrm{p}=0.035)$ and center $(\mathrm{p}<0.001)$ were significantly associated with the placement of a temporization procedure in multivariable regression analysis. 


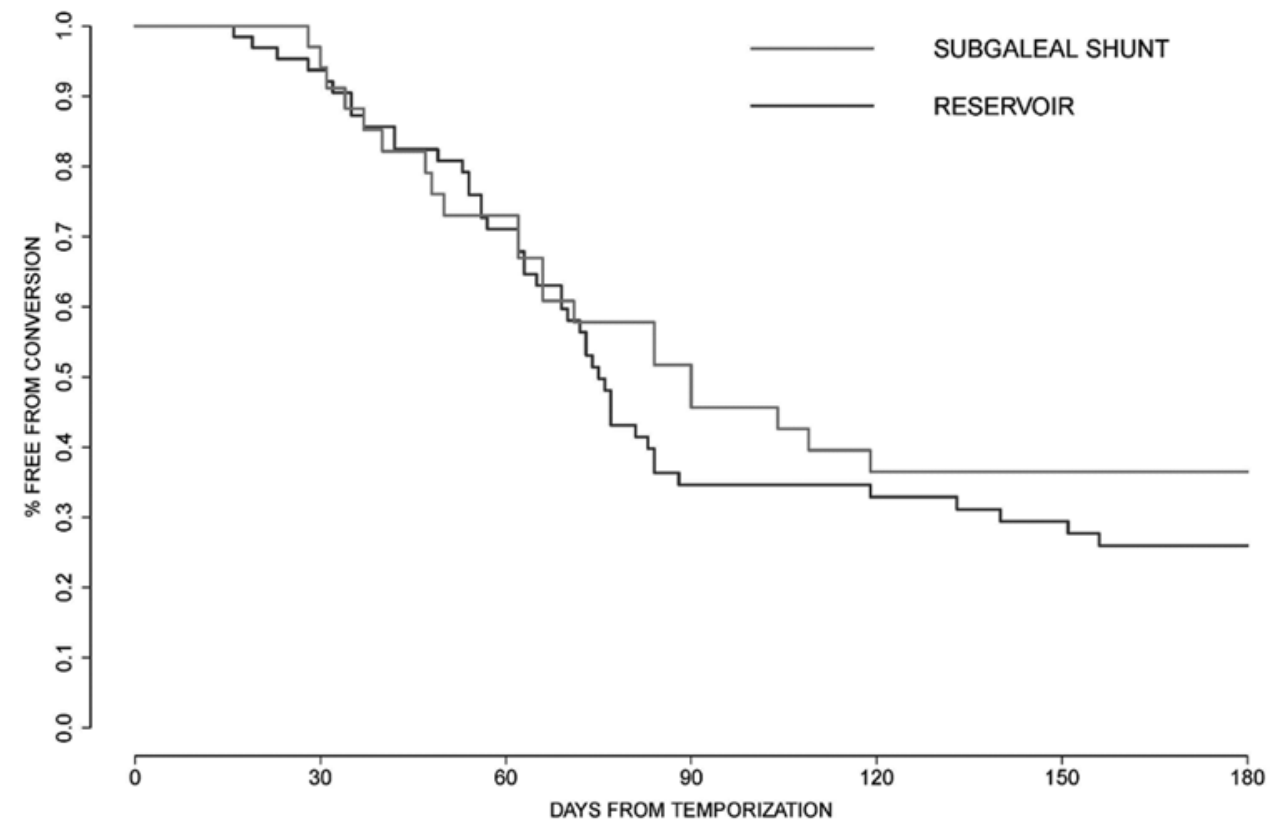

FIG. 6. Line graph showing the rate of conversion to a permanent shunt according to the initial temporization approach.

Larger ventricles $(p<0.002)$ and a full fontanel $(p<0.001)$ predicted the conversion from a temporization device to a permanent CSF shunt. ${ }^{24}$ Importantly, the study identified many significant differences in delivery of care among the centers, including conversion rates from temporization to CSF shunting (25\%-96\%), types of temporization devices used, admission/discharge frequencies per patient, length of stay at the tertiary care center, and the frequency and modalities used for medical imaging of these patients. This dramatic institutional variation underscored the importance of establishing standardized care algorithms and practice guidelines for these medically complex infants.

In the early stages of the study line, it became clear that despite the fact that each participating center was considered a high-volume academic pediatric neurosurgery practice, the patient populations differed among centers, and most importantly, the approaches to management contrasted widely. An 800-g premature infant with a unilateral Grade 3 IVH, for example, would undergo VP shunt placement at 1 center, VSGS placement at another center, reservoir placement for intermittent tapping at yet another, and serial lumbar taps at another. This realization, coupled with the strong HCRN data supporting reduction of shunt infection across the network by standardizing care, led to the hypothesis of "center effect" and the desire to standardize how the surgeons made decisions. ${ }^{12,24}$ The series of rubrics defined in this paper (Fig. 1-4) took 3 years of consensus building to create, based on data from both the first and second paper in the series, ${ }^{24,30}$ and where there were no data, consensus among participating surgeons. For example, there was nearly uniform consensus that split sutures, a bulging fontanelle, or bradycardia should be relevant rather than just the issue of a ventricular FOHR of 0.55 alone (Figs. 1 and 4), and that infants without these findings did not represent those with the disease process under scrutiny. Surprisingly, once decision-making was aligned, it was a straightforward process to turn the proce- dures into checklists for the surgeons to follow and be observed performing correctly. There was no clear consensus on the infant weight at which to convert either VR or VSGS to permanent CSF diversion; therefore, a "surgeon preference" option was built into the decision rubrics. A small, but relevant, finding in this study was that surgeons did tend to use $2000 \mathrm{~g}$ as the cutoff infant weight for both initial temporization and ultimate conversion; therefore, moving forward this cutoff value has been incorporated into the current HCRN protocols for the management of infants with $\mathrm{PHH}$.

As the body of work in this study line began to expand, it became clear that clinical decision-making in infants with PHH is dictated largely by clinical signs of increased intracranial pressure. It was important, then, to determine if surgeons at each center assessed premature infants for these signs in the same way. At each of the HCRN centers, pairs of surgeons independently assessed for split sutures and bulging fontanels, as defined a priori as greater than 2-mm separation of the sagittal suture at the midparietal point and as the fontanel above the level of the surrounding bone, respectively. In the assessment of 38 infants (76 independent observations), the kappa coefficient for split suture was 0.84 (95\% CI 0.67-1.00) and for bulging fontanel was 0.65 (95\% CI 0.41-0.90). ${ }^{29}$ This study confirmed that there was an adequate degree of interrater reliability among HCRN investigators in their assessment of these clinical signs. This ability to consistently determine which patients met criteria for treatment was considered critical in the formation of the current prospective trial.

There were some critical differences in surgeon compliance, particularly when enrollment into the study based on initial ventricular size and physical examination findings were concerned. Once patients were enrolled, however, it appeared that there was no difference within the "Temporization versus Shunt" rubric or the conversion rubrics.

The role of compliance in protocols is complex and 
TABLE 3. Reported complication rates according to initial temporization approach

\begin{tabular}{cccc}
\hline Complication & VSGS $(\mathrm{n}=36)$ & VR $(\mathrm{n}=66)$ & $\mathrm{p}$ Value \\
\hline Infection & $5(14 \%)$ & $11(17 \%)$ & $0.71^{*}$ \\
\hline CSF leak & $2(6 \%)$ & $3(5 \%)$ & $1.0 \dagger$ \\
\hline Death & $6(17 \%)$ & $7(11 \%)$ & $0.54 \dagger$ \\
\hline
\end{tabular}

* Using the Pearson chi-square test.

$\dagger$ Using the Fisher exact test.

the requirements necessary for consideration of surgeon compliance vary from study to study. In the initial HCRN shunt infection quality improvement study, it was noted that the infection trend declined as the compliance gradually rose,,$^{12}$ but there were many variables that could have been responsible for the outcome. The well-noted WHO surgical checklist paper published in 2009 in the New England Journal of Medicine reported that $56.7 \%$ of the patients received treatment that was in full compliance with the protocol overall. Despite this low percentage, there were highly significant findings of reduced morbidity and mortality. ${ }^{10}$ For the multiple-point protocol, prespecified portions of the protocol were chosen and evaluated. The complexity of that protocol may be what reduced compliance overall. It should be noted that the shunting outcomes in posthemorrhagic hydrocephalus (SOPHH) rubrics were considered complex by the HCRN surgeons, yet ultimately served as a valuable roadmap for decisionmaking, and that compliance as determined by surgeon, weight preferences, and conformity to the dictates of the protocol was considered high. This is highly relevant for further network studies in which standardized protocols are a major part, and further effort must be made toward compliance centered on enrollment eligibility.

There are several limitations of this study. Despite its prospective nature, it was not randomized. The study was initially conceived for each center to cross over halfway during an 18-month period (i.e., 9 months) so that all surgeons who performed VR placement at a center would cross over to performing VSGS placement for the latter half of the study. There was, however, significant concern from multiple centers' IRBs such that this was not feasible across the network. The study was therefore redesigned as a prospective cohort study in which the highly statistically relevant "center effect" would be ideally mitigated and the results between procedures would be closer to a true comparative effectiveness study. While this remains the only multicenter study of its kind, it still does not yet fully settle the issue raised in a editorial comment by Dr. Joseph Piatt in $2010^{21}$ in which our field was chastised for continuing to concern itself with "surgeon-centric" outcomes such as infection or shunting rates. While important to patient quality of life, the issue raised in that editorial was relevant in that perhaps ultimately, a parent would prefer a child to be better off neurodevelopmentally, whether a shunt was present or not. This led to the inclusion of the secondary outcome of the BSID-III in this cohort at 18-22 months, which will be reported in a second follow-up paper.
TABLE 4. Compliance with rubrics at various SOPHH time points

\begin{tabular}{lcccc}
\hline \multicolumn{1}{c}{ Rubric } & $\begin{array}{c}\text { Investigator } \\
\text { Surgeons } \\
(\%)\end{array}$ & $\begin{array}{c}\text { Noninvestigator } \\
\text { Surgeons } \\
(\%)\end{array}$ & $\begin{array}{c}\text { Overall (\%) } \\
\text { Value* }\end{array}$ \\
\hline Enrollment & $79 / 87(91)$ & $44 / 58(76)$ & $123 / 145(85)$ & $0.014 \dagger$ \\
\hline Temporization & $56 / 57(98)$ & $44 / 45(98)$ & $100 / 102(98)$ & $1.0 \ddagger$ \\
\hline Conversion & $37 / 39(95)$ & $22 / 27(82)$ & $59 / 66(89)$ & $0.11 \ddagger$ \\
\hline $\begin{array}{l}\text { Combining } \\
\text { data from } \\
\text { above }\end{array}$ & $172 / 183(94)$ & $110 / 130(85)$ & $282 / 313(90)$ & $0.006 \dagger$ \\
\hline
\end{tabular}

* Comparing HCRN surgeons and other surgeons.

$\dagger$ Using the Pearson chi-square test.

$\ddagger$ Using the Fisher exact test.

$\S$ Treating time points for the same patient as separate observations.

\section{Conclusions}

Once the management decisions and surgical techniques used for infants weighing less than 1500 grams with Grade 3 or 4 IVH were standardized across HCRN sites, there was no observed difference in the proportion of infants who ultimately underwent conversion to a permanent shunt from either a VR or a VSGS at 6 months. Compliance was high among both HCRN investigator and noninvestigator surgeons, with differences noted on patient enrollment parameters, but no differences noted once patients were enrolled. Due to the results of this primary study, it does not appear to be necessary to perform a further randomized controlled trial based on shunt conversion alone. Further results on the neurodevelopmental status of this cohort will be forthcoming and will be critical to determining any next necessary steps in the study line.

\section{Acknowledgments}

We would like to thank our colleagues who kindly agreed to participate in this HCRN project and allowed collection of patient data for the purpose of this publication: D. Brockmeyer, M. Walker, R. Bollo, J. Blount, J. Johnston, B. Rocque, L. Ackacpo-Satchivi, P. Dirks, J. Rutka, M. Taylor, D. Curry, R. Dauser, A. Jea, S. Lam, R. Ellenbogen, J. Ojemann, A. Lee, A. Avellino, I. Pollack, S. Greene, E. Tyler-Kabara, T. S. Park, J. Leonard, M. Smyth, N. Tulipan, A. Singhal, and P. Steinbok. In addition, this work would not have been possible without the outstanding support of the dedicated personnel at each clinical site and the DCC. Special thanks goes to N. Tattersall (Salt Lake City), A. Arynchyna (Birmingham), H. Ashrafpour (Toronto), S. Ryan (Houston), A. Anderson (Seattle), K. Diamond (Pittsburgh), D. Mercer (St. Louis), S. Gannon (Nashville), A. Cheong (British Columbia), and M. Langley, N. Nunn, R. Reeder, V. Friemann, and J. Alvey (Utah DCC). Lastly, Amita Bey, MPH, research coordinator at the University of AlabamaBirmingham during the initial years of the study, now Program Director of the Vanderbilt Surgical Outcomes Center for Kids, deserves significant thanks for her work during the initial enrollment and initiation of the network-wide study, as well as Stephen Gannon for more recent technical assistance with the final drafts.

In addition, funding for the completion of the portion of the study included in this paper was received from a National Institute of Neurological Disorders and Stroke (NINDS) ARRA grant (no. 1RC1NS068943-01) and through an agreement signed between the HCRN and the Hydrocephalus Association. No industry funding was used during the completion of this study. 
The HCRN has been funded by NINDS (grant no. 1RC1NS068943-01), the Patient Centered Outcome Research Institute (grant no. CER-1403-13857), the Gerber Foundation (reference no. 1692-3638), private philanthropy, and the Hydrocephalus Association. Dr. Simon was supported by Award K23NS062900 from the NINDS and the Seattle Children's Center for Clinical and Translational Research, and CTSA grant no. ULI RR025014 from the National Center for Research Resources, a component of the NIH.

\section{Appendix HCRN Members}

The HCRN currently consists of the following clinical centers and investigators: Primary Children's Hospital, University of Utah (J. Kestle); Children's Hospital of Alabama, University of Alabama at Birmingham (J. Oakes, C. Rozzelle); Hospital for Sick Children, University of Toronto (J. Drake, A. Kulkarni); Texas Children's Hospital, Baylor College of Medicine (T. Luerssen, W. Whitehead); Seattle Children's Hospital, University of Washington (S. Browd, T. Simon); Children's Hospital of Pittsburgh, University of Pittsburgh (M. Tamber); St. Louis Children's Hospital, Washington University in St. Louis (D. Limbrick); Monroe Carell Jr. Children's Hospital at Vanderbilt, Vanderbilt University Medical Center (J. Wellons, R. Naftel, C. Shannon); British Columbia Children's Hospital, University of British Columbia (P. McDonald); Colorado Children's Hospital (T. Hankinson); Johns Hopkins (E. Ahn); Children's Hospital of Los Angeles (M. Krieger); Nationwide Children's Hospital (J. Pindrick); Alberta Children's Hospital (J. Riva-Cambrin); HCRN DCC, Department of Pediatrics, University of Utah (R. Holubkov); and Alberta Children's Hospital, University of Calgary (J. RivaCambrin).

\section{References}

1. Adams-Chapman I, Hansen NI, Stoll BJ, Higgins R: Neurodevelopmental outcome of extremely low birth weight infants with posthemorrhagic hydrocephalus requiring shunt insertion. Pediatrics 121:e1167-e1177, 2008

2. Brandt CA, Nadkarni P, Marenco L, Karras BT, Lu C, Schacter L, et al: Reengineering a database for clinical trials management: lessons for system architects. Control Clin Trials 21:440-461, 2000

3. Brouwer A, Groenendaal F, van Haastert IL, Rademaker K, Hanlo P, de Vries L: Neurodevelopmental outcome of preterm infants with severe intraventricular hemorrhage and therapy for post-hemorrhagic ventricular dilatation. J Pediatr 152:648-654, 2008

4. Brouwer AJ, Brouwer MJ, Groenendaal F, Benders MJ, Whitelaw A, de Vries LS: European perspective on the diagnosis and treatment of posthaemorrhagic ventricular dilatation. Arch Dis Child Fetal Neonatal Ed 97:F50-F55, 2012

5. Brouwer AJ, Groenendaal F, van den Hoogen A, VerboonMaciolek M, Hanlo P, Rademaker KJ, et al: Incidence of infections of ventricular reservoirs in the treatment of posthaemorrhagic ventricular dilatation: a retrospective study (1992-2003). Arch Dis Child Fetal Neonatal Ed 92:F41F43, 2007

6. Cooke R, Rennie J, Weindling M, Whitelaw A, Stewart A, Hope $\mathrm{P}$, et al: Randomised trial of early tapping in neonatal posthaemorrhagic ventricular dilatation. Arch Dis Child 65:3-10, 1990

7. de Vries LS, Liem KD, van Dijk K, Smit BJ, Sie L, Rademaker KJ, et al: Early versus late treatment of posthaemorrhagic ventricular dilatation: results of a retrospective study from five neonatal intensive care units in The Netherlands. Acta Paediatr 91:212-217, 2002

8. Fulmer BB, Grabb PA, Oakes WJ, Mapstone TB: Neonatal ventriculosubgaleal shunts. Neurosurgery 47:80-84, 2000
9. Gaskill SJ, Marlin AE, Rivera S: The subcutaneous ventricular reservoir: an effective treatment for posthemorrhagic hydrocephalus. Childs Nerv Syst 4:291-295, 1988

10. Haynes AB, Weiser TG, Berry WR, Lipsitz SR, Breizat AH, Dellinger EP, et al: A surgical safety checklist to reduce morbidity and mortality in a global population. N Engl J Med 360:491-499, 2009

11. Jian L, Hang-song S, Zheng-lang L, Li-sheng Y, Heng W, $\mathrm{Nu} \mathrm{Z}$ : Implantation of Ommaya reservoir in extremely low weight premature infants with posthemorrhagic hydrocephalus: a cautious option. Childs Nerv Syst 28:1687-1691, 2012

12. Kestle JR, Riva-Cambrin J, Wellons JC III, Kulkarni AV, Whitehead WE, Walker ML, et al: A standardized protocol to reduce cerebrospinal fluid shunt infection: the Hydrocephalus Clinical Research Network Quality Improvement Initiative. J Neurosurg Pediatr 8:22-29, 2011

13. Levy ML, Masri LS, McComb JG: Outcome for preterm infants with germinal matrix hemorrhage and progressive hydrocephalus. Neurosurgery 41:1111-1118, 1997

14. Limbrick DD Jr, Mathur A, Johnston JM, Munro R, Sagar J, Inder T, et al: Neurosurgical treatment of progressive posthemorrhagic ventricular dilation in preterm infants: a 10-year single-institution study. J Neurosurg Pediatr 6:224-230, 2010

15. Mathews TJ, Miniño AM, Osterman MJ, Strobino DM, Guyer B: Annual summary of vital statistics: 2008. Pediatrics 127:146-157, 2011

16. McComb JG, Ramos AD, Platzker AC, Henderson DJ, Segall HD: Management of hydrocephalus secondary to intraventricular hemorrhage in the preterm infant with a subcutaneous ventricular catheter reservoir. Neurosurgery 13:295300, 1983

17. Mihajlović M, Mrdak M, Radlović V, Nikolić I, Rakić M, Repac N, et al: Application of the Ommaya reservoir in the treatment of hydrocephalus in prematurely born children: correlation with animal results. Srp Arh Celok Lek 141:812,2013

18. Nadkarni PM, Brandt C, Frawley S, Sayward FG, Einbinder $\mathrm{R}$, Zelterman $\mathrm{D}$, et al: Managing attribute-value clinical trials data using the ACT/DB client-server database system. J Am Med Inform Assoc 5:139-151, 1998

19. Nagy A, Bognar L, Pataki I, Barta Z, Novak L: Ventriculosubgaleal shunt in the treatment of posthemorrhagic and postinfectious hydrocephalus of premature infants. Childs Nerv Syst 29:413-418, 2013

20. Peretta P, Ragazzi P, Carlino CF, Gaglini P, Cinalli G: The role of Ommaya reservoir and endoscopic third ventriculostomy in the management of post-hemorrhagic hydrocephalus of prematurity. Childs Nerv Syst 23:765-771, 2007

21. Piatt JH: Editorial. Posthemorrhagic ventricular dilation. J Neurosurg Pediatr 6:222-223, 2010

22. Rahman S, Teo C, Morris W, Lao D, Boop FA: Ventriculosubgaleal shunt: a treatment option for progressive posthemorrhagic hydrocephalus. Childs Nerv Syst 11:650-654, 1995

23. Richard E, Cinalli G, Assis D, Pierre-Kahn A, Lacaze-Masmonteil T: Treatment of post-haemorrhage ventricular dilatation with an Ommaya's reservoir: management and outcome of 64 preterm infants. Childs Nerv Syst 17:334-340, 2001

24. Riva-Cambrin J, Shannon CN, Holubkov R, Whitehead WE, Kulkarni AV, Drake J, et al: Center effect and other factors influencing temporization and shunting of cerebrospinal fluid in preterm infants with intraventricular hemorrhage. J Neurosurg Pediatr 9:473-481, 2012

25. Rizvi SA, Wood M: Ventriculosubgaleal shunting for posthaemorrhagic hydrocephalus in premature neonates. Pediatr Neurosurg 46:335-339, 2010

26. Savitz MH, Malis LI: Subgaleal shunting: a 20-year experience. Neurosurg Focus 9(6):ecp1, 2000

27. Tubbs RS, Smyth MD, Wellons JC III, Blount J, Grabb PA, 
Oakes WJ: Life expectancy of ventriculosubgaleal shunt revisions. Pediatr Neurosurg 38:244-246, 2003

28. Wang JY, Amin AG, Jallo GI, Ahn ES: Ventricular reservoir versus ventriculosubgaleal shunt for posthemorrhagic hydrocephalus in preterm infants: infection risks and ventriculoperitoneal shunt rate. J Neurosurg Pediatr 14:447-454, 2014

29. Wellons JC III, Holubkov R, Browd SR, Riva-Cambrin J, Whitehead W, Kestle J, et al: The assessment of bulging fontanel and splitting of sutures in premature infants: an interrater reliability study by the Hydrocephalus Clinical Research Network. J Neurosurg Pediatr 11:12-14, 2013

30. Wellons JC III, Shannon CN, Kulkarni AV, Simon TD, RivaCambrin J, Whitehead WE, et al: A multicenter retrospective comparison of conversion from temporary to permanent cerebrospinal fluid diversion in very low birth weight infants with posthemorrhagic hydrocephalus. J Neurosurg Pediatr 4:50-55, 2009

31. Whitelaw A: Repeated lumbar or ventricular punctures in newborns with intraventricular hemorrhage. Cochrane Database Syst Rev (1):CD000216, 2001

32. Whitelaw A, Kennedy CR, Brion LP: Diuretic therapy for newborn infants with posthemorrhagic ventricular dilatation. Cochrane Database Syst Rev (2):CD002270, 2001

33. Willis B, Javalkar V, Vannemreddy P, Caldito G, Matsuyama J, Guthikonda B, et al: Ventricular reservoirs and ventriculoperitoneal shunts for premature infants with posthemorrhagic hydrocephalus: an institutional experience. J Neurosurg Pediatr 3:94-100, 2009

\section{Disclosures}

Dr. Limbrick has received support of non-study-related clinical or research effort from Medtronic and Storz. Dr. Browd is cofounder and has equity and stock options in Aqueduct Neurosciences, Inc., Aqueduct Critical Care, Inc., and Navisonics, Inc., all of which are unrelated to the current study. None of the sponsors participated in design and conduct of this study; collection, man- agement, analysis, and interpretation of the data; or preparation, review, or approval of this manuscript. Its contents are solely the responsibility of the authors and do not necessarily represent the official view of the sponsors.

\section{Author Contributions}

Conception and design: Wellons, Kestle. Acquisition of data: Wellons, Shannon, Riva-Cambrin, Kulkarni, Limbrick, Whitehead, Browd, Rozzelle, Simon, Tamber, Oakes, Drake, Luerssen, Kestle. Analysis and interpretation of data: Wellons, Shannon, Holubkov. Drafting the article: Wellons, Shannon, Holubkov. Critically revising the article: Wellons, Holubkov, Kestle. Reviewed submitted version of manuscript: all authors. Approved the final version of the manuscript on behalf of all authors: Wellons. Statistical analysis: Holubkov. Administrative/technical/material support: Wellons. Study supervision: Wellons, Riva-Cambrin, Kulkarni, Limbrick, Whitehead, Browd, Rozzelle, Simon, Tamber, Oakes, Drake, Luerssen, Kestle.

\section{Supplemental Information}

\section{Online-Only Content}

Supplemental material is available with the online version of the article.

Online Appendix. https://thejns.org/doi/suppl/10.3171/2017.1. PEDS16496.

\section{Previous Presentations}

Results from this study have been presented in oral form at the AANS/CNS Pediatric Section Annual Meeting in Toronto, ON, Canada, in December 2013, and in poster form at the AANS/CNS Pediatric Section Annual Meeting in Amelia Island, Florida, in December 2014

\section{Correspondence}

John C. Wellons III, Department of Neurosurgery, Vanderbilt University Medical Center, 2200 Children's Way, DOT9226, Nashville, TN 37232. email: jay.wellons@vanderbilt.edu. 